\title{
Pratiques Traditionnelles de Conservation de la Nature à L'épreuve des Faits Chez Les Peuples Riverains de la Réserve de Biosphère de Yangambi (RDC)
}

\author{
Justin Kyale Koy \\ Doctorant, Université de Kisangani, Faculté de sciences Sociales, \\ Administratives et Politiques, Département de Sciences Politiques et \\ Administratives, Projet FCCC, RDC, Kisangani.

\section{Alphonse Maindo Monga Ngonga} \\ Professeur, Université de Kisangani, Faculté de sciences Sociales, \\ Administratives et Politiques, Département de Sciences Politiques et \\ Administratives, Directeur national de Tropenbos International-RDC, RDC, \\ Kisangani.
}

\section{doi: 10.19044/esj.2017.v13n8p328 URL:http://dx.doi.org/10.19044/esj.2017.v13n8p328}

\begin{abstract}
The Riparian people of the Biosphere Reserve of Yangambi (Turumbu and Bamanga) have traditionally developed practices of nature conservation. However, the ongoing socio-cultural changes in the region are likely to affect the sustainability of those practices. On one hand, this study aims to identify the Turumbu and Bamanga's existing traditional practices and knowledge about conservation. On the other hand, it aims to analyze the socio-cultural changes on their sustainability. Data collection techniques comprised of historical archives exploitation, focus-group, and individual interviews conducted for 20 villages surrounding the Biosphere Reserve of Yangambi (BRY). Investigations revealed a huge range of practices affecting plant communities and wildlife species as well as natural phenomena restricting either hunting or fishing activities. Findings also pointed out traditional knowledge and mystical-based considerations from agricultural practices. In addition, cemeteries, sites for the rites of passage, the foodbased taboos, and legendary sites were target to sacralisation. The grafting between religion, colonization, modernity and culture, resulted to a bottleneck which affects such practices. In spite of these constraints, the study shows that there are still opportunities at the multilevel (local, national, global) for their reactivation.
\end{abstract}


Keywords: Traditional practices, sacred spaces, nature conservation, Biosphere Reserve of Yangambi, Democratic Republic of Congo.

\section{Résumé}

Les peuples riverains de la Réserve de Biosphère de Yangambi (Turumbu et Bamanga) ont développé dans leurs traditions respectives, des pratiques liées à la conservation de la nature. Cependant, face aux mutations socioculturelles qui s’opèrent dans la région, leur pérennisation est sujette au doute. Cette étude vise d'une part à répertorier les pratiques et savoirs traditionnels de conservation existant chez les Turumbu et Bamanga, et d'autre part, à analyser l'impact des mutations socioculturelles sur leur pérennisation. Les techniques utilisées pour récolter les informations sont fondées sur la consultation des archives historiques, l'animation des focus group et des interviews individuelles dans 20 villages environnant la Réserve de Biosphère de Yangambi (RBY). Les investigations ont permis de découvrir un vaste champ desdites pratiques, touchant aux espèces floristiques et fauniques ainsi qu’aux phénomènes naturels limitant l'activité de chasse et/ou de pêche. Il en a été constaté les savoirs et considérations mystiques dans les pratiques agricoles. En outre, les cimetières, les espaces de rites d'initiation culturelle, les interdits alimentaires et les espaces légendaires ont aussi joui de la sacralisation. Le greffage entre la religion, la colonisation, la modernité, la culture et les réalités pratiques s’est érigé en un goulot d'étranglement, réduisant par ricochet, la dimension conservatoire desdites pratiques. En dépit de ces contraintes, les opportunités s’offrent au niveau tant local, national que mondial pour leur réactivation.

Mots-clés: Pratiques traditionnelles, espaces sacrés, conservation de la nature, Réserve de Biosphère de Yangambi, République Démocratique du Congo.

\section{Introduction}

À partir de 1980, l'importance des savoirs locaux est prise en compte dans les débats mondiaux sur la nature (Roué, 2012; Tubiana, 2005). Cela a ouvert la voie aux chercheurs de diverses disciplines scientifiques qui en ont trouvé un champ d'investigation (Sene et al., 2013; Renard et al., 2013; Savadogo et al., 2011; Pinton \& Grenand, 2007; Garcia et al., 2006; Beaulaton \& Roussel, 2005; Sow, 2003; Beaulaton, 1999). L’intégration des pratiques traditionnelles dans le processus de gestion moderne des aires protégées ou de la biodiversité pourrait en constituer une stratégie efficace et durable (Ehinnou Koutchika et al., 2014). À ce sujet, Garcia et al. (2006) notent que «les bois sacrés sont considérés comme une institution alliant conservation de l'environnement et respect des valeurs endogènes». Dans le 
même ordre d’idées, Roussel (2005) démontre la durabilité des savoirs naturalistes locaux en soulignant « qu'appartenir à une tradition est considéré comme la garantie d'une certaine ancienneté et si les éléments de la biodiversité concernés sont parvenus jusqu’à nous, c'est que l'utilisation qui en est faite est nécessairement « durable ». En Côte d’Ivoire par exemple, la religion traditionnelle des Ehotilé a conduit, sous l'angle de cogestion des ressources naturelles, à la création du Parc national des îles Ehotilé (Malan Djah, 2009). Un peu partout en Afrique, les communautés rurales ont, depuis la nuit de temps, sacralisé certains espaces forestiers dotés de divers interdits pour faciliter l'organisation d'une diversité d'activités culturelles (Yameogo, 2015). Sous cette même perspective, quelques essences floristiques et fauniques ont été mises à l'abri de toute exploitation au regard du rôle qu’elles jouent dans le domaine du sacré ou de la pharmacopée. Aussi, les ruraux africains ont développé dans leurs perceptions, une série de savoirs locaux protégeant leurs activités de survie, concourant par ricochet, à la conservation des forêts. Cependant, comparativement à l'Afrique de l'Ouest (Bénin, Togo, Côte d’Ivoire, Gabon, Niger, Guinée, Sénégal, Burkina Faso, etc.) où la littérature scientifique marque une présence dans cette thématique, en République Démocratique du Congo (RDC) par contre, elle accuse un certain déficit, en dépit de l'existence desdites pratiques au sein des communautés locales (CL). La diversité des bois sacrés, le rôle joué par les pratiques traditionnelles dans l'encadrement des pratiques locales de survie et les facteurs socioculturels influant sur leur décadence y restent faiblement documentés. En outre, les approches modernes de conservation de la nature, introduites pendant la colonisation belge, n'auraient pas intégré cette dimension locale en vue d'associer les peuples autochtones dans le processus de création et de gestion des aires protégées. La logique guidée par l’ordre colonial était liée aux considérations «préservationnistes » au détriment d'une vision « dévolutioniste», pour reprendre les expressions de Boissière et Doumenge (2008). Par ailleurs, si le terme d’aire protégée est forgé par l'occident, il n'en demeure pas moins que les communautés congolaises disposaient des leurs sous d'autres dénominations. Les Bamanga et les Turumbu, riverains de la réserve de biosphère de Yangambi (RBY) en RDC ne dérogent pas à la règle. En effet, ces derniers ont concouru à la protection de la nature dans leur milieu grâce aux pratiques traditionnelles héritées de leurs coutumes. Ainsi ont-ils développé ces pratiques dans l'exercice de certaines activités culturelles ou de survie, notamment l'initiation à la coutume, la circoncision, la chasse, la pêche et l'agriculture. Bien d'espèces fauniques et floristiques y ont fait l'objet d'interdits divers, décourageant en revanche, leur exploitation. Toutefois, depuis quelques décennies passées, ces pratiques traversent une zone de turbulence rendant difficile, leur pérennisation dans le temps. Or si aujourd'hui, la tendance mondiale est 
donc de conserver les pratiques locales, de réguler leur utilisation et de les valoriser, cela suppose donc de les identifier pour en dresser l'inventaire et de mettre en place des dispositifs de conservation et de suivi, tel que le notent Louafi et Roussel (2005). C'est à ce niveau qu'il sied de cerner l'importance d'analyser lesdites pratiques chez les peuples riverains de la RBY en vue d'orienter leur intégration dans les politiques publiques relatives à la conservation de la nature. Cette étude vise ainsi d'une part à répertorier les savoirs et pratiques traditionnels de conservation de la nature existant chez les Turumbu et Bamanga, et d'autre part, à analyser l'impact des mutations socioculturelles sur leur pérennisation. Comment les peuples riverains de la RBY ont-ils conservé la nature dans leurs pratiques traditionnelles? Quels sont les facteurs influençant le déclin desdites pratiques? Telles sont les questions autour desquelles gravite cette recherche. L’étude présente d'abord le panorama des pratiques traditionnelles de conservation de la nature. Les facteurs explicatifs de l'effondrement desdites pratiques sont ensuite étayés avant de présenter les opportunités liées à leur réactivation.

\section{Approche Méthodologique}

\section{Milieu d'étude et richesse de la biodiversité à conserver dans la RBY}

L’étude a été réalisée chez les peuples riverains de la RBY. Celle-ci est située à environ $100 \mathrm{Km}$ et $62 \mathrm{Km}$, respectivement à l'Ouest et au Nord de la ville de Kisangani en RDC (figure 1). D’une superficie légale évaluée à 225.000 hectares [(Institut pour l'Étude Agronomique du Congo Belge (INEAC), 1939)], ses coordonnées géographiques sont comprises entre $24^{\circ} 18^{\prime}$ et $25^{\circ} 08^{\prime}$ de longitudes Est et $00^{\circ} 43^{\prime}$ et $01^{\circ} 08^{\prime}$ de latitudes Nord, avec des altitudes variant entre 400 et 500m. Située dans la zone équatoriale, elle connaît un climat du type Af de Koppen (Kombele, 2004; Beguin, 1958; Van Wambeke \& Evrard, 1954) et reçoit une moyenne annuelle de 1.750 $\mathrm{mm}$ de pluies, auxquelles se joint une température moyenne de $24,9^{\circ} \mathrm{C}$ (Kombele, 2004). La réserve est arrosée sur toute son étendue par des ruisseaux et rivières se jetant soit sur le Fleuve Congo au sud-ouest, soit sur la rivière Aruwimi au Nord, soit encore sur la rivière Lindi à l'Est. Le milieu a fait l'objet des études floristiques et agronomiques réalisées à l'époque coloniale par l'INEAC. À Yangambi-centre, siège dudit institut, l'on rencontre un herbarium composé de 15.000 sortes de plantes (la plus grande collection d'Afrique centrale), un xylarium (collection de bois tropicaux), une bibliothèque, des plantations de café, de cacao, de bananes et de caoutchouc, un complexe agro-industriel, des parcelles expérimentales d'arbres, des laboratoires de recherche faiblement équipés, etc. (Hiergens, 2010). La biodiversité de la forêt tropicale présente une richesse d'arbres inégalée d'environ 32.000 sortes (Hiergens, 2010). Donis (1956) y a 
dénombré 128 essences forestières susceptibles de fournir du bois d'œuvre grâce à leur grande dimension. S'agissant de la faune aquatique, Gosse (1963) en a dénombré 239 espèces. Cependant, ces chiffres sont sujets au doute et nécessitent des études approfondies pour leur mis à jour, comme l’ont souligné Poll et Gosse (1963). Son environnement humain est constitué majoritairement des peuples Turumbu et Bamanga (revendiquant le monopole de propriété coutumière des terres) et minoritairement des groupes ethniques allochtones rencontrés généralement à Yangambi-centre. C’est auprès de ces deux premiers groupes ethniques que les données ont été récoltées. Ces derniers sont profondément attachés à la forêt. Celle-ci constitue la principale source de revenu pour eux. Elle est aussi une source de pharmacopée, eu égard à l'insuffisance des institutions sanitaires dans le milieu et à la perception des communautés sur l'incurabilité de certaines maladies par la médecine moderne.

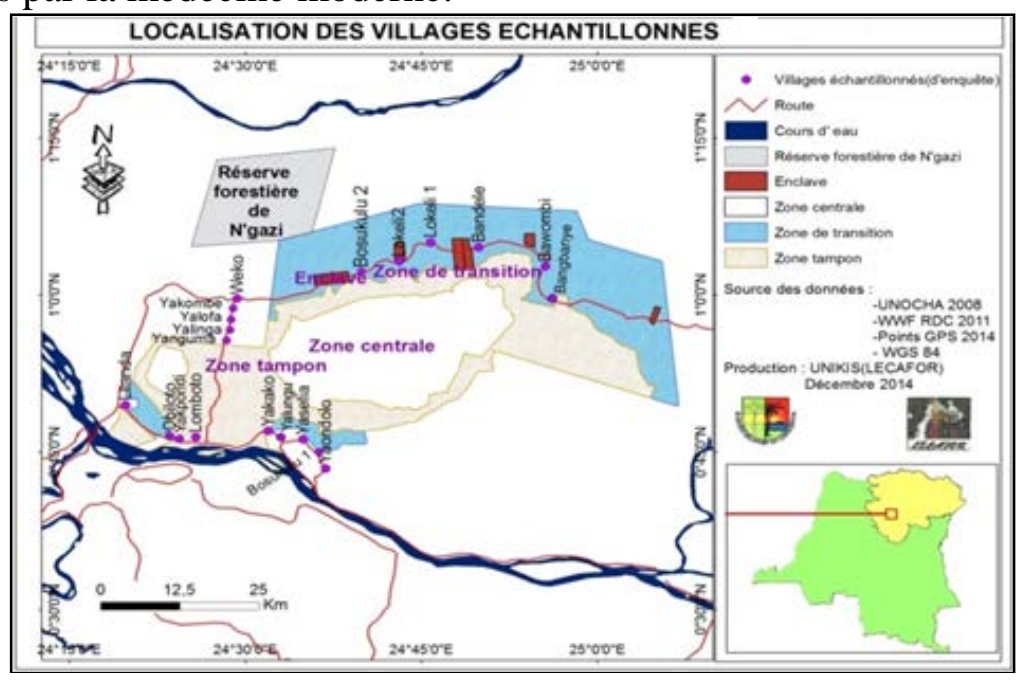

Figure 1. Localisation de la zone d'étude

\section{Collecte Des Données}

La collecte des données relatives à l'objet de cette étude a été faite en mobilisant trois principales techniques. Il s'agit notamment de la documentation (constituée essentiellement des archives historiques), des focus group et des interviews individuelles. Les documents d'archives ont été consultés au Musée Royal d'Afrique centrale (MRAC), aux Archives africaines du Ministère Belge des Affaires Étrangères, commerce extérieur et coopération au développement, au Centre de recherche de l'Institut National pour l'Étude et la recherche agronomiques au Congo (INERA) à Yangambi ainsi qu'à la Division provinciale de l'intérieur, affaires coutumières et sécurité à Kisangani. Les sources documentaires (anciennes) sont précieuses dans les études portant sur les savoirs et pratiques locaux, comme le souligne 
Marchenay (2005).Les informations collectées dans ces archives, en raison de leur valeur historique, ont été triangulées à travers les mémoires collectives des communautés étudiées et les interviews individuelles. Les focus group, entendus au sens de Gavard-Perret et al. (2011) et Moreau et al. (2004) ont été administrés aux communautés pour comprendre leur perception sur les pratiques traditionnelles de conservation de la nature. La pertinence des focus group dans cette étude se justifie, comme le souligne Crosnier (2005), par le fait que « les savoirs et pratiques locaux sont transmis par la tradition orale et façonnés dans un contexte historique et culturel donné, possédant une forte dimension symbolique et sociale ». En outre, l'apport de cette technique dans les études qualitatives est, tel que le note Touré (2010) « de permettre aux chercheurs de répondre à des questions relatives à des phénomènes sociaux, de comprendre ces phénomènes tels qu'ils sont vécus et perçus et d'agir sur eux dans une perspective de changement social ». Ils ont été animés aux mois de février, mars, avril et mai 2015 avec les notables, les chefs locaux, les élites locales et les sages des villages. La disposition sous forme de cercle a été adoptée pour éviter des considérations hiérarchiques entravant la liberté d'expression entre le chercheur (animateur) et les participants (Moreau et al., 2004; Gavard-Perret et al., 2011). Afin de susciter une dynamique productive de groupe dans chacun de ces focus group, le nombre des participants était compris entre six et douze en moyenne, tel que recommande Touré (2010). Un effort a été fourni pour intégrer les jeunes, les adultes et les vieillards dans le groupe. L’intégration de toutes ces couches sociales était motivée d'une part par la recherche de compréhension de la perception des communautés sur les pratiques traditionnelles en étude, et d'autre part, par le partage de façon indirecte des connaissances y afférentes aux membres du groupe qui en étaient ignorants. Ce dernier aspect rencontre le point de vue de Moreau et al. (2004) soutenant que «les échanges favorisent l'émergence des connaissances, opinions et expériences comme une réaction en chaîne ».Les grands axes des échanges étaient centrés sur les aspects de conservation relatifs à l'usage desdites pratiques dans les domaines de la chasse, de la pêche, de l'agriculture, des rites coutumiers, des interdits divers ou des contes légendaires et des facteurs inhibant leur consolidation. À l'issue de la confrontation libre des points de vue, les compromis liés à l'objet d'étude étaient retenus dans chacun des thèmes débattus. Néanmoins, la diversité d’opinions lors des échanges avait joué un rôle non négligeable dans la compréhension du degré d'intérêt et de connaissance qu'avaient les membres des groupes sur lesdits thèmes. Les interviews individuelles ont permis de recueillir les informations auprès des vieillards et autres personnes-clés dont le déplacement pour faire partie des focus group posait problème. Eu égard à la pertinence des informations culturelles qu'ils détenaient, ils ont constitué 
des personnes-ressources indispensables à cette recherche. La sélection des villages constituant l'échantillon géographique (Esiso Asia Amani, 2012) était fondée sur le critère de proximité qu’ont ces entités avec la RBY (Figure 1). Le site étant entouré d'une centaine des villages, les focus group n’ont été réalisés que dans 20. Parmi ceux-ci, l’on note chez les Bamanga, les villages Bangbanye, Bawombi, Bakobi, Lokeli I et Lokeli II. Du côté des Turumbu, les villages concernés par l'étude étaient Bosukulu II, Wekodjialapanda, Yakombe, Yalofa, Yalinga, Yanguma, Yakpondi, Obiloto, Lilanda, Yakako I, Yalungu, Yaseli, Bosukulu I et Yaondolo II. En vue de faciliter l'identification de quelques espèces floristiques citées lors des investigations, les noms de ces dernières ont été notés en langues locales et leur traduction était faite par deux botanistes appartenant chacun aux communautés étudiées. Le recours à ces trois principales sources de données décrites ci-haut se justifie par le souci de vouloir valider les connaissances par leur triangulation, eu égard aux limites que connaît chacune d'entre elles. La combinaison des sources de données correspond bien, comme le souligne Trudel (2002), à une conception courante de la pratique ethnohistorique. En effet, il se constate souvent dans quelques documents d'archives, des contradictions alimentées par la tradition orale qui ne remonte pas assez loin et reste difficilement transmissible dans le temps (Vansina, 1987; Marmitte, 1922). À titre illustratif, l'enquête ethnographique réalisée par Lauwers (1932a, 1932b) à deux phases dans deux clans Turumbu (Yawalo et KombeOtole) a donné deux versions contradictoires de leur appartenance au peuple Yawenda. Aussi, l'incompréhension des langues entre les interlocuteurs aurait posé problème de fidélité dans la description des faits par l'homme blanc. En dépit de ces limites, les sources orales représentent un dispositif essentiel pour appréhender le passé des peuples n’ayant pas connu l'écriture (Baillargeon, 1993; Grenand, 1980; Halbwachs, 1997). L’analyse qualitative de contenu a été faite pour comprendre la pertinence des informations récoltées dans les archives, les focus group ou les corpus oraux. Les données recueillies et analysées ont ainsi produit des résultats présentés au point suivant.

\section{Résultats De Recherche}

Tel qu’il a été souligné précédemment, les pratiques traditionnelles de conservation de la nature chez les Turumbu et les Bamanga sont axées sur plusieurs domaines. Les points suivants les décrivent avant de relever les facteurs favorisant leur déclin sous une perspective discursive. 
Interdire de toucher la plante et observer les astres pour rentabiliser l'exploitation des ressources fauniques

La chasse et la pêche constituent des activités de survie anciennement pratiquées par les Turumbu et les Bamanga. Étant des moyens permettant à ces peuples de se procurer du revenu, ces derniers ont puisé dans le monde du sacré, quelques interdits en vue de rentabiliser lesdites activités. En effet, dans leur incursion forestière, il est interdit aux chasseurs et pêcheurs Bamanga de toucher à l'arbre de « desia ${ }^{53}$ »(Afraegle paniculata). Dans le dialecte Bamanga, « desia » est traduit littéralement par la non-atteinte de l'objectif poursuivi. Aussi, pendant la période de pleine lune, les Turumbu et les Bamanga observaient une certaine indifférence vis-à-vis de la chasse et de la pêche nocturne ${ }^{54}$. La chasse des rats et autres petits rongeurs était plus concernée par cette indifférence. Pour ces communautés, l’éclairage de la lune permet aux animaux sauvages et aux poissons de se rendre compte des pièges, hameçons ou filets qui leur sont tendus. Cela a contribué à une sorte de régulation culturelle de l'activité. La conséquence découlant de nonobservance de ces interdictions est la faible capture des gibiers ou des poissons.

\section{Conserver la nature dans les pratiques agricoles}

Comme chez la plupart des peuples ruraux de la RDC, l'agriculture est la principale activité de survie chez les Turumbu et les Bamanga. Ainsi, dans ce domaine, quelques essences forestières sont interdites d'être coupées dans les champs de cultures grâce au rôle qu'elles jouent dans la production des chenilles et de bois d'œuvre ou artisanat local, la protection des cultures contre le vent et des esprits maléfiques ${ }^{55}$.Concernant la production des chenilles, la conservation touche aux essences forestières telles que Pertianthus macrocarpus, Erythrophleum suaveolens et Entandrophrgma sp. En outre, Prioria balsamifera et Entandrophrgma sp., servant à la fabrication des pirogues sont aussi mis à l'abri de coupe. Le Pericopsis elata est protégé par ces peuples grâce à sa valeur économique dans la production du bois d'œuvre. Dans leurs champs, les paysans gardent les arbres ayant un houppier faible, ceux protégeant les cultures contre le vent et ceux ayant des considérations mystiques ou coutumières. Parmi les arbres supposés être porteurs de mauvais esprits, les Bamanga ont cité le Ngobu (Autranella congolensis). En effet, la tradition orale ${ }^{56}$ raconte que pendant les anciennes guerres que ce peuple a connues avec les autres groupes ethniques, les ancêtres se cachaient dans la cavité de cet arbre. Au fur du temps, l'arbre a

\footnotetext{
${ }^{53}$ Focus group des mois de février et mars 2015.

${ }^{54}$ Focus group des mois de février, mars et avril 2015.

${ }^{55}$ Focus group des mois de février, mars et avril 2015.

${ }^{56}$ Bonga Michel, Interview, Bawombi, février 2015.
} 
été à la base de beaucoup d'accidents mortels dans les champs de cultures lors de son abattage. Remémorant l'histoire, les Bamanga ont expliqué ces accidents en soulignant que les esprits des ancêtres se cacheraient dans cet arbre. D'où la crainte de l'abattre lors de défrichement. Ces arbres non abattus dans les champs jouent un autre rôle (secondaire) d'identification des jachères ou des limites de celles-ci. Ils appartiennent aux propriétaires des champs conformément à la coutume locale et à la loi ${ }^{57}$.

Une autre pratique traditionnelle qui a concouru non seulement à la protection des forêts mais aussi à la fertilisation du sol chez les Turumbu et Bamanga reste l'abandon des champs de cultures en jachère ( « mbizo » chez les Bamanga, " nduu » chez les Turumbu) pendant une longue période d'environ 20 ans. De l'avis de ces peuples, une telle durée favoriserait la production de manioc et debanane plantain. Intégrant ce savoir local dans la politique de paysannerie mise sur pied dans la région à partir de 1942, l'INEAC a fixé la durée de jachère entre 15 et 16ans (Muller \& Vervier, 1953). Toutefois, cette durée a sensiblement diminué jusqu'à atteindre une moyenne de 3,4 ans ${ }^{58}$. Cette moyenne est incluse dans la fourchette de 3 à 6 ans selon Kombele (2004). Les jachères situées non loin des villages présentent une faible durée, en dépit de l'exposition des cultures aux menaces des animaux domestiques.

\section{Les cimetières: sacrés pour les anciens Turumbu, banalisés pour les Bamanga}

Dans certaines communautés africaines, les cimetières font parties des sites sacrés conservés des générations en générations (Kigongo, 2009; Kouami Kokou \& Sokpon, 2006). En RDC, ils constituent les lieux de rencontre entre les vivants et les morts. De ce fait, ils jouissent de la sacralisation pour beaucoup de communautés rurales. C'est le cas des peuples Turumbu. En effet, si les cimetières (Mbilà) chez les Turumbu étaient sacrés, il n'en était pas le cas chez les Bamanga où les « totè » se trouvaient devant les cases. Dans la culture ancienne chez les Turumbu, les morts étaient enterrés au fond du village. Les tombes faisaient l'objet d'entretien et les arbres ou les forêts qui les entouraient bénéficiaient de la protection. Toutefois, l'objectif pour eux n'était pas de conserver les forêts mais plutôt les cimetières. La conservation n'était qu'une conséquence de la sacralisation du lieu, comme l'a noté aussi Juhé-Beaulaton (1999) dans l'ancienne Côte des Esclaves (située actuellement au Sud du Togo et du Bénin). Par contre chez les Bamanga, enterrer les morts loin des cases était

\footnotetext{
${ }^{57}$ RDC (2002). «Loi n 011/2002 du 29 août 2002 portant code forestier », Journal officiel de la $R D C, 43^{\text {ème }}$ Année, Numéro Spécial, Cabinet du Président de la République, Kinshasa.

${ }^{58}$ Focus group des mois de février et mars 2015.
} 
perçu comme un manque d'affection vis-à-vis des défunts ${ }^{59}$. Ceux-ci étaient et sont généralement inhumés devant leurs cases. Les habits qu’ils portaient étaient posés au-dessus de leurs tombes pour faciliter leur identification. Cet enterrement devant les cases éloignait toute possibilité d’oublier la mémoire des défunts.

\section{Protéger la nature dans les espaces des rites traditionnels et par les interdits divers}

Certains espaces forestiers étaient aménagés par les peuples riverains de la RBY pour des raisons rituelles. Ainsi, les « lobè » ou « ebolo » chez les Turumbu ou les «Mambela » chez les Bamanga qui constituaient des espaces forestiers d'initiation et des rites culturels auraient été conservés dans leur état. D’après Van Dieren (1933) et Brandt (1917), les non-initiés, notamment les enfants et les femmes ne pouvaient pas assister, ni fréquenter la forêt de "Mambela ». Il était strictement interdit de cultiver dans ces espaces. Les grandes décisions engageant le village y étaient prises. C'est notamment le cas de résolution des conflits opposant les membres du même village ou des villages voisins.

Dans le domaine de la circoncision («mkafil » ou « mboïge » chez les Bamanga), une forêt spéciale était aménagée dont l’accès au public (hommes et femmes) était ouvert (Van Dieren, 1933). Cependant, en tant que site sacré, cette forêt ne subissait pas de pression liée à l'agriculture. En termes d'interdits alimentaires, l'homme qui venait d'être circoncis ne pourrait pas manger la peau de l'éléphant, la viande de cynocéphale, de chimpanzé, de singe, de python, de tortue et les chenilles, pendant trois ans après la circoncision (Van Dieren, 1933). Le risque était de voir sa plaie devenir incurable. Toutefois, une certaine régulation coutumière consistant à organiser une cérémonie de pardon s’observait en cas de transgression de cette coutume ${ }^{60}$. L'on est ici en face de ce que Savadogo et al. (2011) qualifient de «sacrifices de désenvoûtement ».Concernant les non-circoncis, la consommation des escargots était interdite pour ne pas compliquer la circoncision. Leur perception était que, comme l'escargot se replie dans sa coquille lorsqu'on le touche, de la même façon, la membrane qui entoure l’organe génital de l'homme peut se rétrécir au moment de la circoncision. En outre, il était interdit aux Bamanga riverains de consommer la viande des animaux aquatiques et de toutes les espèces de serpents, au risque de voir leurs peaux devenir comme celles de ces espèces animales ${ }^{61}$. Toujours chez les Bamanga, la consommation des espèces de poisson capturées en dehors de leurs sites habituels ou à des périodes non-habituelles était interdite à

\footnotetext{
${ }^{59}$ Focus group des mois de février et mars 2015.

${ }^{60}$ Focus group des mois de février et mars 2015.

${ }^{61}$ Focus group des mois de février et mars 2015.
} 
certaines personnes n’ayant pas satisfait les critères liés à l’âge, au sexe, au nombre d'enfants ou de génération ${ }^{62}$. Ainsi, chaque site était identifié par les poissons qui s’y trouveraient. De même, les pêcheurs connaissaient les poissons capturés pendant la nuit et ceux capturés pendant la journée. C’est lorsqu'il y a une capture hors du commun qu'une telle interdiction était évoquée. De ce fait, la capture des poissons dépendait de leur consommation par le pêcheur. Ce dernier n'hésitait pas à remettre dans l'eau, toute espèce de poisson qu'il ne peut consommer. Dans la même logique, d'autres interdictions frappaient les enfants enceintes, les maris de ces derniers et les enfants $^{63}$. Ainsi une femme enceinte se réservait de manger la viande de porc-épic (au risque de voir l'enfant naître avec des galles et blessures sur la peau) et le « poisson électrique » (au risque d'avorter). Aussi, la femme dont la grossesse n'a pas encore totalisé trois mois et son mari se gardaient de manger le premier gibier abattu par le chasseur dans une campagne de chasse ou les premiers poissons capturés par le pêcheur recourant à la nasse comme technique de pêche. Une telle interdiction frappait également les enfants dont les dents menaçaient de pousser. Le fondement de cette interdiction était que le chasseur ou le pêcheur concerné se voyait son activité devenir improductive en cas de non-respect.

Chez les Turumbu de Yelongo, la consommation de python n'est pas autorisée par la coutume. La tradition orale ${ }^{64}$ raconte que Botike, l’aîné de la famille avait abattu ce serpent dans la forêt pendant que son père Elongo était gravement malade. Dès son retour au village, il trouva ses frères en train de pleurer leur père. Un conflit de choix s'est créé entre l'organisation d'une part de deuil du père, et d'autre part, de la cérémonie coutumière sur le partage de python. La première option étant privilégiée, s'en est suivi l'abandon de python durant la période des obsèques. Ce dernier étant décomposé, sa consommation a été interdite à toute la communauté. Pour d'autres clans des groupements du secteur Turumbu (Yawenda, Yambau et Weko), les enfants et les femmes ne mangeaient pas la viande de python et de léopard (Demptine, SD). Les conséquences qui découlaient du nonrespect de ces interdits étaient nombreuses. Elles allaient de la maladie à la mort. En outre, le Ricinodendron heudelotii (« lisongo » chez les Turumbu) sur lequel on suspendait le python pour organiser des cérémonies ancestrales avant de le disséquer et distribuer ses parties aux différents clans du village restait à l'abri de toute coupe chez les Turumbu. De même, le palmier à huile auquel le chef du village avait l'habitude de recourir pour ses libations ne pourrait pas être déraciné, ni coupé après sa mort (Demptine, SD). En revanche, un de ses frères s'en servait pour tirer du vin de palme (sans

\footnotetext{
${ }^{62}$ Focus group des mois de février et mars 2015.

${ }^{63}$ Focus group des mois de février et mars 2015.

${ }^{64}$ Basanga, J.-P., Interview, Yakombe, mars 2015.
} 
toutefois abattre l'arbre) dont une quantité était versée sur la tombe et une autre consommée par les frères et sœurs du défunt. Chez les deux communautés en étude, l'arbre dénommé « $\mathrm{zulo}^{65}$ " ou "akusawanga ou lototo ${ }^{66} "$ (Fernandoa adolfi-friderici) est connu pour sa considération mystique $^{67}$. Son écorce sert de fétiche pour les chasseurs et les pêcheurs qui veulent rentabiliser leur activité. Elle est aussi utilisée par les charlatans ou guérisseurs pour soigner les maladies de leurs clients. Les humains qui veulent arracher son écorce y vont en respectant une série de conditions. Ils ne portent pas les habits et préparent un billet symbolique de monnaie à déposer sous son pied. En outre, l'instrument ayant servi d'arrachage de son écorce devrait être déposé sous son pied. Au retour, ils devraient se garder de regarder en arrière jusqu'à parcourir une certaine distance. Dans l'hypothèse où ces conditions ne sont pas observées, l'homme se voit être touché par la démangeaison susceptible d'entraîner des blessures énormes au corps.

Non loin de Yangambi-centre, l'on rencontre le Ceiba pentandra qui reste conservé, eu égard au mythe qui lui est collé. Les noms attribués à cet arbre traduisent son caractère mythique: "arbre du Roi », "arbre de l'authenticité », «baobab ». L'appellation de "l'arbre du Roi » tirerait son origine de la visite du Roi des Belges à l'époque coloniale. Ce mythe se renforce aussi par sa dimension touristique et sa grandeur. En effet, il est difficile d'accueillir un étranger à Yangambi-centre pendant deux semaines sans qu'il s'y soit rendu (Figure 2). Il constitue ainsi un site potentiellement touristique du milieu.

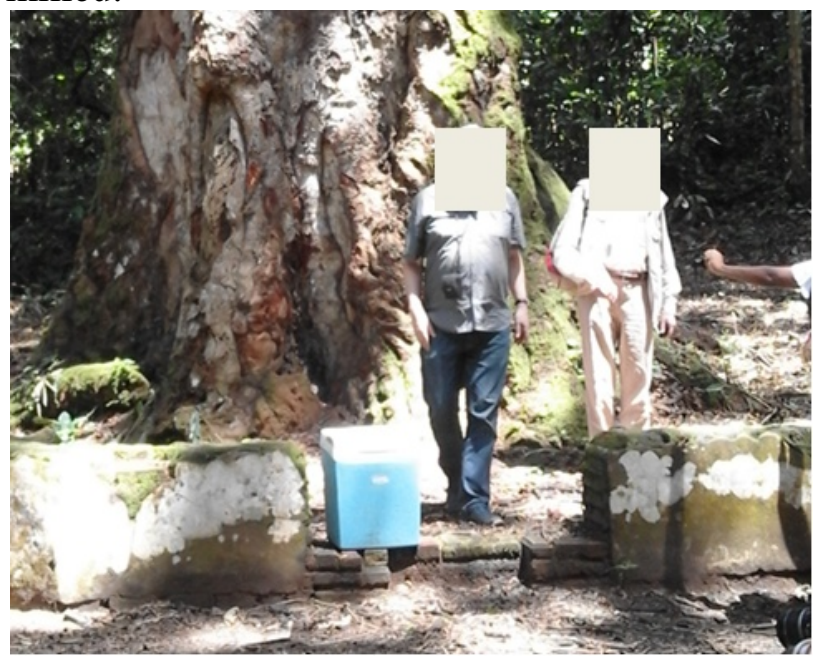

Figure. 2. Arbre dit de l'authenticité de la RBY. Source: Photo prise par Justin Kyale Koy en mai 2016

\footnotetext{
${ }^{65}$ En dialecte Bamanga.

${ }^{66}$ En dialecte Turumbu.

${ }^{67}$ Focus group des mois de février, mars et avril 2015.
} 


\section{Conserver les espaces par des faits légendaires}

Les contes légendaires remémorés par les peuples Bamanga des villages Bandele et Yalibonga ont joué un rôle non négligeable dans la protection des forêts environnant les chutes de la rivière Lobilo. Leurs ancêtres auraient construit une maison dont la hauteur atteignit le ciel ${ }^{68}$. Un jour, le vent violent aurait renversé cette maison, tuant ainsi bon nombre d'entre eux. Les survivants se seraient dispersés. Depuis longtemps, l'endroit est resté faiblement fréquentable. Les bruits occasionnés par l'activité humaine à cet endroit entraineraient de fortes pluies. Aussi, au niveau des chutes, l'on rencontre des grottes dont la disposition est semblable aux chaises bien arrangées. Cet emplacement des grottes (Figure 3) est interprété par les habitants de ces villages comme le lieu de repos de mauvais esprits et des ancêtres ${ }^{69}$.
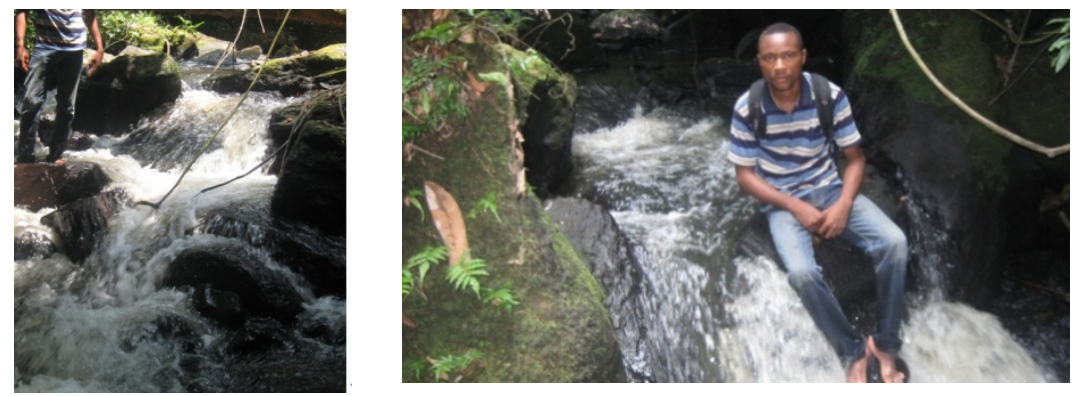

Figure 3. Grottes des chutes de la rivière Lobilo. Source : photo prise par les auteurs en mars 2015

Aussi, à l'époque de l’INEAC, les blancs fréquentaient régulièrement ces chutes ${ }^{70}$. Généralement, ils y seraient accompagnés des noirs qui, malheureusement auraient été interdits (par l'homme blanc) d'y arriver. Pour les autochtones, ces blancs y partaient pour se procurer de la magie issue des esprits des ancêtres. Ce qui justifierait l'interdiction des noirs pendant que les blancs visitaient les grottes. Un charlatan du nom de Bébé Rico qui a visité ces grottes serait rentré de là très abattu par les esprits ${ }^{71}$. Tous ces faits ont imprimé dans le chef de ce peuple, la peur de fréquenter les cavités des grottes situées autour desdites chutes. Lors des investigations menées, la visite de ces chutes et cavités des grottes a été strictement interdite par le chef du village Bandele. Cependant, après plusieurs supplications, ledit chef a fini par accorder l'autorisation en nous faisant accompagner par l'un de ses

\footnotetext{
${ }^{68}$ Focus group du mois de mars 2015.

${ }^{69}$ Amoti Nganga Mopero, Chef du village Bandele, Interview, Bandele, mars 2015.

${ }^{70}$ Focus group du mois de mars 2015.

${ }^{71}$ Focus group du mois de mars 2015.
} 
agents. Une tendance à la démystification de ces chutes est constatée ces derniers jours avec la présence des exploitants miniers non loin du site.

Les différentes pratiques traditionnelles ci-haut décrites ont structuré la perception des Turumbu et Bamanga sur le rapport homme-nature dans le temps. Elles sont remémorées à travers la tradition orale et les pratiques réelles sur le terrain à des degrés divers. Les unes sont d'actualité, les autres par contre se sont effondrées au regard de diverses contraintes culturelles, religieuses, coloniales, modernes et pratiques qu'il sied de relever dans la discussion des résultats.

\section{Discussion Des Résultats}

La consolidation des pratiques traditionnelles de conservation de la nature chez les Turumbu et Bamanga reste mitigée, eu égard aux multiples mutations observées dans le milieu. Ainsi, l'essentiel de ce point tourne autour des facteurs concourant au déclin des pratiques traditionnelles ou des savoirs locaux liés à la conservation de la nature chez ces peuples riverains de la RBY.

Controverse entre religion, colonisation, modernité, culture et réalités pratiques autour des pratiques traditionnelles de conservation de la nature

L'avènement du christianisme dans la région de Yangambi et de Bengamisa a bouleversé le fonctionnement de plusieurs pratiques traditionnelles locales. Ces peuples s’y sont attachés depuis l'époque coloniale (qui en constitue un véritable vecteur d'expansion du christianisme en RDC). La présence d'anciennes missions évangéliques à Yangambi, Yakusu, Bengamisa et Isangi en démontre sans peine. Dans presque chaque village où les investigations ont été réalisées, l’on rencontre au moins une confession religieuse. Le recours à certaines croyances locales est prêché par les « hommes de Dieu » comme une pratique contraire à l'ordre divin. C'est notamment le cas d'interdits alimentaires divers, de circoncision traditionnelle et d'initiations rituelles. La religion traditionnelle a ainsi cédé la place au christianisme. L’érosion des pratiques traditionnelles par les nouvelles religions a été également constatée au Bénin où les fêtes de " sortie » de nouvelles récoltes qui constituaient jadis des pratiques communautaires qui ont permis de conserver certaines variétés des plantes se sont effondrées avec l'avènement des religions monothéistes (Baco, 2007). De même, la pratique animiste de la société Mossi et les sites sacrés vodun rencontrés respectivement au Burkina Faso et au Bénin ont connu une certaine régression avec la poussée des religions importées comme l’islam et le christianisme (Savadogo et al., 2011; Juhé-Beaulaton, 2003). JuhéBeaulaton (2003) a en outre souligné qu’au Bénin, « certains prêtres célébraient des offices dans des forêts sacrées pour démontrer à la population 
la puissance supérieure de leur dieu unique ». Sous cette perspective, Somé (2002) souligne que:

«L'idéologie civilisatrice du $19^{\text {è }}$ siècle, par ses constructions de pensée négatives sur l'Afrique et par l'échelle des valeurs qu'elle a établie entre les sociétés humaines, a abouti à l’infériorisation du Noir et à son rejet au faubourg de l'histoire. [...] les racistes occidentaux de l'époque d’impérialisme colonial faisaient remarquer qu'il fallait dissocier le christianisme de la civilisation occidentale et utiliser cette religion comme moyen de faire accepter aux noirs leur situation d'arriération».

Le christianisme a œuvré dans la région des Bamanga et Turumbu, comme un peu partout en RDC, sous la bénédiction du pouvoir colonial. Les communautés autochtones étaient ainsi affaiblies dans leur entreprise religieuse (traditionnelle) par la persuasion du christianisme et la coercition des pouvoirs publics. Ceci corrobore avec le point de vue de Juhé-Beaulaton (2003) qui soutient « qu’après la conquête coloniale, les politiques menées par les administrateurs contribuèrent elles aussi à la déstructuration du tissu social et de l'espace ». Les adeptes de «Kitawala », l'une des sectes religieuses revendiquant l'émancipation de l'homme noir, ont connu de sévères surveillances et répressions pendant la période coloniale (Gilot, 1947; Colinet, 1942a; Colinet, 1942b; Schollar, 1944; Cote, 1939), voire peu après cette époque (Province orientale, Arrêté n²21/10 du 13 février 1962 interdisant la secte « Kitawala ». Lesdits adeptes étaient soit relégués, soit emprisonnés (De Valck, 1938 ; Coenen, 1937). Une publication faite par Bossche (1954) a même recensé au profit du service de sécurité de la colonie, toutes les associations et sectes traditionnelles défendant les valeurs traditionnelles. Face à un tel climat de psychose généralisée, les pratiques traditionnelles ne faisaient que s'effondrer.

Pour ce qui est de diminution de la durée des jachères, trois principaux facteurs y sont évoqués par les paysans lors des investigations sur le terrain. Il s’agit de la démographie galopante, du chômage et de l'introduction des cultures nouvelles et de nouvelles habitudes culturales. Pour ce qui est de la croissance démographique dans le milieu, les chiffres sont impressionnants si on les compare dans une période d'environ 82 ans. En effet, la population du Secteur Turumbu est passée de 9.569 en 1933 (Rapport Affaires indigènes et main-d'œuvre (AIMO), 1933) à 25.154 en 1984 (INS, 1984), puis à 79.098 en 2015 (Secteur Turumbu, 2015). En 1933, le nombre d'habitants du Secteur Bamanga était d'environ 17.380 (Rapport AIMO, 1933) alors qu'en 1984, ce nombre a été estimé à 43.605 (INS, 1984) lors du recensement scientifique, puis à 110.154 habitants en 2015 (Secteur Bamanga, 2015). Dans une société où l'on sait que les jachères font partie de l'héritage familial, il est difficile que lesdites jachères partagées entre les fils 
d'un même père connaissent une longue durée. La pression démographique a été aussi identifiée par Kouami Kokou et Sokpon (2006) comme l'un des facteurs de déclin des pratiques locales de gestion forestière au Bénin et au Togo. Sous l'angle du chômage, il sied de noter qu'avant l'accession de la RDC à la souveraineté nationale, beaucoup d'autochtones non-cultivateurs Turumbu étaient engagés à l'INEAC (Laurent, 1937; Libert, 1936; Steens, 1934; Rapport AIMO, 1934). Avec la baisse des activités de ce centre de recherche après l'indépendance, une diminution sensible du personnel a été constatée (Hiergens, 2010). Le recours à l'agriculture constitue dès lors, une des stratégies de survie pour ces peuples, y compris ceux qui travaillent en vue de compenser le déficit du revenu salarial. En outre, les paysans de la région de Yangambi étaient regroupés en paysannat et coopérative (Henry, 1979; Jurion \& Henry, 1967; Staner, 1955; Muller \& Vervier, 1953; Malengreau, 1952; Henry, 1951). La non-consolidation de ces politiques rurales après la colonisation belge a entraîné comme conséquence, l'abandon du paysan. Chacun pouvait cultiver là où il veut et quand il veut sans respecter la durée de jachère. Aussi, dans le passé lointain, ces peuples cultivaient pour le ventre. Il s'agissait d'une agriculture exclusivement de subsistance. Avec l'introduction de nouvelles cultures comme le riz, le niébé, ...une visée quasiment productiviste a été constatée, même si le délabrement des routes continue à les décourager. Ces nouvelles cultures sont beaucoup adaptées aux jachères soulignent les paysans. De ce fait, il est tout à fait logique que la durée de ces dernières devienne courte. C'est ici l'occasion de soutenir le point de vue de Baco et al. (2007) selon lequel, « les nouvelles habitudes alimentaires, les innovations agricoles constituent des facteurs qui contribuent aussi à l'érosion de l'agrobiodiversité et des savoir-faire s'y rapportant ». Aussi, Alphandéry et Fortier (2005) soulignent «qu'enfermés dans un cadre opposant tradition et modernité, les savoirs paysans devenaient de ce fait illégitimes, comme s'ils appartenaient à un monde clos et immobile ». En plus, chez les Turumbu, la pratique dite « zongisa », traduite littéralement par revenir sur ou replanter les cultures après la récolte, prend de plus en plus de l'ampleur comme l'ont constaté également Kombele (2004) et Alonga (2013). Cela réduit davantage ladite durée et appauvrit le sol. Cette pratique mérite d'être inscrite dans une perspective des mutations locales menaçant l'agriculture, comme le soulignent Baco et al. (2007). Les écosystèmes forestiers de la RBY restent ainsi exposés à la surexploitation dans l'avenir, eu égard à l'appauvrissement du sol. Les paysans finiront par s'y rabattre pour trouver des espaces fonciers fertiles. Un autre facteur ayant influencé le déclin des pratiques traditionnelles reste la sorcellerie. Celle-ci, répandue dans le milieu, a découragé les vivants à enterrer les défunts au 
fond du village chez les Turumbu. Loma Mbula ${ }^{72}$, Chef de Groupement Yawenda note à ce sujet que « enterrer loin des maisons, son proche suspecté d'avoir été ensorcelé, offrirait une occasion aux sorciers de récupérer le cadavre. Le souhait est d'organiser l'inhumation devant la case en vue d'assurer la surveillance continue de la tombe. Ceci contribue à la disparition progressive des cimetières regroupés généralement au bout des villages ${ }^{73}$. Sous l'angle pratique, quelques-uns des savoirs et croyances locaux ont du mal à contribuer à la protection de la nature. Dans le contexte d'une agriculture itinérante sur brûlis par exemple, il est difficile de garder les essences remplissant des fonctions socioéconomiques. Le feu endommage souvent ces arbres, en dépit de la volonté qu'ont les paysans à les conserver. En outre, la circoncision accompagnée des cérémonies coutumières s'effondre au profit de la médecine moderne. Les parents amènent leurs enfants aux institutions sanitaires pour les faire circonscrire afin de les épargner de nombreux risques d'infections dont le tétanos. Cet état de choses paraitrait logique dans la mesure, le caractère rudimentaire des instruments utilisés ne pouvait qu'exposer les personnes à circoncire à de tels dangers. Aussi, interdire une communauté avide d'aliments à manger telle ou telle autre espèce faunique reste une question délicate. Au moment où les forêts et les eaux étaient riches en faune sauvage, le choix entre ce qui est interdit et ce qui ne l'est pas ne poserait pas problème. Le pêcheur ou le chasseur pouvait facilement se garder d'abattre telle espèce faunique, sachant que la capture d'une autre était possible. En plus, l'élevage, peu développé d'ailleurs, n'offre pas des produits destinés habituellement à la consommation locale. Il vise plus la résolution des problèmes auxquels les paysans sont confrontés. C'est le cas de viol, des arrestations, d'accueil des visiteurs ou des cérémonies de festivité. À cela s'ajoute l'échange de cultures entre les communautés qui a modifié profondément la perception des Turumbu et Bamanga sur les interdits coutumiers. En effet, il est difficile de rencontrer les villages où les membres appartiennent tous à une même tribu ou une même ethnie. Les migrations des peuples, l'esclavagisme local, les alliances matrimoniales et la quête des ressources naturelles ont mis sur pied, les villages «hétéroculturels ». Devant une telle situation, des échanges culturels peuvent jouer en faveur ou en défaveur des unes ou des autres coutumes. Ce qui est sacré chez certains peuples ne l'est pas nécessairement chez les autres. Tel que le notent les Bamanga «les allochtones noncirconcis qui se sont retrouvés chez nous n'auraient pas nécessairement respecté la coutume locale. Leur circoncision n'ayant pas posé problème, cela a attiré notre curiosité et conduit par ricochet à la banalisation des

${ }^{72}$ Loma Mbula, Interview, Bosukulu I, avril 2015.

${ }^{73}$ Loma Mbula, Intervieuw, Bosukulu I, avril 2015. 
interdits alimentaires relatifs à la circoncision ${ }^{74}$ ». Dans le même ordre d'idées, Baco et al. (2007) notent que le bouleversement des habitudes alimentaires est imputable aux brassages entre communautés venant d'horizon divers (nord et sud du pays).Par ailleurs, avec une visée économique des activités, mêmes les communautés sont interdites de consommer une espèce faunique, son abattage reste de pratique. C'est ainsi que les serpents sont vendus aux allochtones par les Bamanga, alors que certains d'entre eux ne les consomment pas. Il y a donc lieu « d'interculturaliser» ces pratiques afin de les rendre opérationnelles dans un monde rural en mutation continue. Sous une autre perspective, certaines des pratiques traditionnelles ci-haut décrites étaient fondées sur des bases éphémères et éloignées de la réalité. En effet, la perception liée à la présence de mauvais esprits sur les arbres n’est pas une réalité spécifique chez les Bamanga. Au Burkina Faso par exemple, elle existe à travers ce que Savadogo et al. (2011) nomment " des lieux hantés », c'est-à-dire les bois supposés abriter des esprits maléfiques. Cependant, si l'on analyse les points de vue des Bamanga sur Autranella congolensis sous l'angle «d'anatomie du bois ", l'on se rend compte que les accidents qu'il provoque sont dus à la cavité se trouvant généralement dans cet arbre. Lors de l'abattage, la chute intervient brusquement, une fois que la hache atteint la cavité sans que le paysan ne s'y attende. Ces accidents deviennent fréquents à cause des techniques rudimentaires auxquelles les paysans recourent pour abattre les arbres de grandes dimensions (Figure 4).

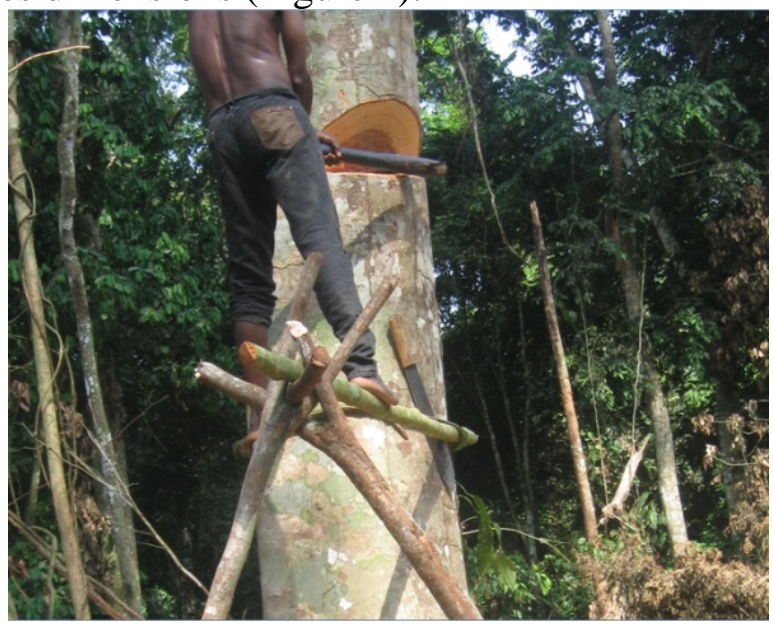

Figure 4. Technique d'abattage de l'arbre dans le champ d'un paysan du village Bandele. Source : photo prise par les auteurs en mars 2015

${ }^{74}$ Focus group des mois de février et mars 2015. 
De même, interdire aux personnes de toucher ou de manger une espèce faunique ne pourrait faire l'objet d'observance que lorsque l'on se retrouve dans un milieu culturellement homogène. Chose difficile dans le contexte actuel où, comme il a été souligné précédemment, de fortes mutations culturelles s’opèrent dans le monde rural. En plus, l'interdiction de toucher à l'espèce dite Afraegle paniculata dans le but de rendre la chasse ou la pêche rentable reste une pratique problématique et éloignée des perspectives conservatoires. Comment peut-on conserver une espèce pour détruire des milliers? Cette question relève de l'éthique dans la conservation de la nature. C'est ainsi que Baco et al. (2007) notent « qu'il existe des bonnes et des mauvaises pratiques, qu'elles soient traditionnelles ou modernes ».

Par ailleurs, il y a lieu de noter que la sacralisation des espèces animales et les interdits qui l'accompagnent sont souvent liés aux considérations discriminatoires qui dominent le monde rural. Cette discrimination se fait souvent au profit des adultes ou des vieillards. Cela est dû à la forte considération coutumière dont bénéficiaient ces espèces animales. Les grandes cérémonies rituelles réunissant les sages et notables des villages avant de disséquer ces animaux (léopard et python par exemple) attestent d'une telle considération. Déjà dans ces cérémonies, les allochtones, les enfants et les femmes étaient excluent. Il est prévisible qu'ils soient aussi exclus de la consommation. Généralement, la viande interdite à une catégorie des personnes est souvent appréciée et savoureuse. Aussi, l'aspect physique de l'arbre ou de la bête et ses effets sur le corps humain ou les autres végétaux le prédisposent à la sacralisation. C'est le cas de Fernandoa adolfifriderici. La dimension mystique de cet arbre est fondée, selon la perception des communautés, sur ses effets sur les humains (la démangeaison) et les autres espèces végétales. Son identification dans un écosystème forestier se constaterait par l'absence des sous-bois et des autres arbres à son côté. Il sied aussi d'ajouter les différents avantages que les hommes peuvent tirer de l'arbre ou de l'animal. Le rapport entre la spiritualité des plantes et leur rôle ou aspect physique a été étayé dans les travaux de Koni Muluwa et Bostoen (2008a, 2008b).

Pour tout dire, il sied de noter qu'en dépit des contraintes relevées précédemment, les opportunités offertes par les enjeux environnementaux de l'heure se présentent et méritent d'être soulignées avant de conclure cette recherche.

\section{Diverses opportunités de réactivation des pratiques traditionnelles en étude}

La tendance à l'effondrement des pratiques traditionnelles liées à la protection de la nature n'interdit pas de penser à leur réactualisation, eu égard aux enjeux environnementaux de l'heure. C'est sous cette perspective 
que Baco et al. (2007) notent que « les pratiques paysannes qui étaient jadis perçues comme catastrophiques et donc combattues sont actuellement acceptées et encouragées ». Dans le même ordre d’idées, Roussel (2005) souligne ce qui suit :

«Pour la communauté internationale qui se préoccupe de gestion de l'environnement et de conservation de la biodiversité, l'intérêt pour les usages de la nature n'est pas nouveau comme l'attestent par exemple les actions de programmes internationaux tel Man and Biosphere de l'Unesco qui, depuis les années soixante-dix, prend en compte, dans ses « réserves de biosphère », les pratiques des populations riveraines. Les savoir-faire ruraux longtemps méprisés par la science officielle, vilipendés par la technique triomphante et considérés même comme la plus importante des causes de dégradation, sont maintenant réhabilités, voire survalorisés ».

Du local au national, de ce dernier au mondial, les opportunités s'offrent dans le domaine de valorisation des pratiques traditionnelles en RDC en général et dans le milieu de cette étude en particulier. Au niveau local, la tradition n'a pas totalement disparu dans le chef de ces communautés. Elle est remémorée par ces dernières et oriente certaines de leurs activités. Le recours à la médecine traditionnelle, aux charlatans, aux cultes des ancêtres et à la sorcellerie traduit l'idée d'un attachement des communautés en étude à la tradition. Aussi, chez les Bamanga de Yambuya, la secte dénommée Kitawala commence à autoriser l'enterrement des morts dans des cimetières localisés au bout des villages. En outre, chez les communautés voisines, une certaine réapparition des pratiques traditionnelles est observée. C'est le cas de «Lilwa » qui prend de nouveau de l'ampleur chez les Bambole. Les Turumbu et les Bamanga pourraient eux aussi emboiter le pas pour réactiver les leurs. Au niveau national, la loi reconnaît la coutume locale et les savoirs traditionnels pour autant que ceux-ci lui soient conformes et ne préjudicient pas l'ordre public. Cette reconnaissance est même consacrée dans l'alinéa 43 de l'article deux de la loi n 14/003 du 11 février 2014 relative à la conservation de la nature qui définit les savoirs traditionnels comme « un ensemble de connaissances, savoir-faire et représentations des communautés locales ayant une longue histoire avec les milieux naturels en matière de conservation et d'utilisation durable de la diversité biologique ». Le processus d'aménagement des forêts, la réglementation protège les sites et les arbres sacrés situés même dans une concession forestière régulièrement attribuée aux personnes physiques ou morales. Dans le même ordre d’idées, en vue de matérialiser la volonté du constituant de la constitution du 18 février 2006 sur l'autorité coutumière, la loi $n^{\circ}$ 015/15 fixant le statut des chefs coutumiers a été prise en 2015. Les communautés locales occupent légalement une place de choix dans l'arsenal 
juridique (national) ayant trait à la gestion des ressources naturelles en RDC. Au niveau mondial, la prise en compte des savoirs locaux et/ou traditionnels dans les politiques environnementales (Roué, 2012) reste une opportunité indéniable dans ce domaine. L'implication de la population locale dans la gestion des écosystèmes devient une nécessité impérieuse et, est à la base des conventions et des stratégies mondiales de conservation de l'environnement (Savadogo et al., 2011).

Des rencontres mondiales et des conventions internationales ont d'ailleurs traité de cette thématique, eu égard à son importance dans la conservation de la biodiversité ${ }^{75}$. Avec les interventions des partenaires mondiaux dans la gestion de la RBY, l'aménagement de certains sites sacrés notamment les chutes de Lobilo pourrait être envisagé un jour en raison de sa potentialité touristique.

\section{Conclusion}

Comprendre, s'adapter et intégrer les visions du monde, les croyances, les systèmes de représentation, les besoins, les intérêts, les pratiques traditionnelles ou les significations des espaces et des ressources appartenant à la population locale sont aussi des conditions pour la réussite du projet de conservation de la nature, note Boya Busquet (2006). La tendance mondiale actuelle est, comme le soulignent Bérard et al. (2005), " de conserver les pratiques locales, de réguler l'utilisation qui en est faite et de les valoriser ».La conservation de la nature était et demeure encore au cœur de quelques pratiques traditionnelles des peuples Turumbu et Bamanga. Le champ desdites pratiques est large. Il touche aux espèces et phénomènes naturels limitant l'activité de chasse et/ou de pêche, aux savoirs locaux et aux considérations mystiques dans les pratiques agricoles, au rôle des cimetières, aux rites d'initiation culturelle, aux interdits alimentaires et aux contes légendaires. La sacralisation des espèces fauniques et floristiques ainsi que les interdits qui l'accompagnent sont souvent liés aux considérations discriminatoires qui dominent le monde rural, à l'aspect physique desdites espèces et leurs effets sur le corps humain ou les autres végétaux et aux avantages que les hommes peuvent tirer de leur usage ou conservation. Cependant, face au greffage d'une part de la tradition avec la

\footnotetext{
${ }^{75}$ L'on peut citer le sommet de Rio de Janeiro en 1992 qui a reconnu la nécessité de protéger les bois sacrés; le colloque de l’Unesco en 1998 consacré aux bois sacrés, à la diversité culturelle et biologique ; l'atelier organisé à Ouagadougou du 18 au 21 juin 2001 sur le thème «Pratiques culturelles, sauvegarde et conservation de la biodiversité en Afrique de 1' Ouest et du Centre »; la Convention concernant la protection du patrimoine mondial, culturel et naturel en 1972; la Convention sur la diversité biologique en 1992; la Convention pour la sauvegarde du patrimoine culturel immatériel de l'humanité en 2003 et la Déclaration des droits des peuples autochtones en 2007.
} 
modernité, et d'autre part, des cultures des communautés de la région, la pérennisation de ces pratiques est actuellement sujette au doute. À cela s'ajoute le poids de la colonisation et du christianisme qui, très tôt a remis en question, le recours aux valeurs traditionnelles du milieu. Ces contraintes ont occasionné la disparition de quelques-unes pendant que d'autres rencontrent de difficultés pour se pérenniser. Par ailleurs, il y a lieu de noter que quelques pratiques répertoriées dans cette étude sont à considérer avec réserve quant à ce qui est de leur apport dans la conservation de la nature. Tel que l’a souligné Dupré (1991), « Il faut se garder du fétichisme qui doterait les savoirs paysans de toutes les vertus qui leur étaient refusées autrefois : ils ne sont pas une panacée universelle propre à résoudre toutes les misères ». Sous cette perspective, Baco et al. (2007) renchérissent en ces termes : «L’argument le plus souvent avancé pour louer sans réserve les mérites des savoirs des sociétés paysannes est qu’ils résultent d'une longue expérience du milieu et qu'ils représentent une adaptation parfaite à leur environnement. Mais c'est oublier que les sociétés migrantes changent d'environnement de même que l'environnement d'une société se modifie sans cesse ». En dépit de l'existence de ces contraintes, les enjeux locaux, nationaux et mondiaux offrent ainsi une opportunité pour la réactivation de celles jugées pertinentes et adaptées aux mutations sociétales de l’heure.

\section{Remerciements}

Les auteurs témoignent de leur gratitude à l’Union Européenne pour les fonds alloués à la recherche doctorale de Justin Kyale Koy; au Centre international de recherche forestière (CIFOR) et à l'Université de Kisangani (UNIKIS) pour l'encadrement pédagogique de ce dernier. Le bureau d'études «RSD» mérite être remercié pour l'efficacité de sa gestion logistique durant la période de collecte des données par Justin Kyale Koy. Henry Badjoko et Pascal Kombe Masiya sont également remerciés pour avoir aidé les auteurs à identifier les noms scientifiques des espèces forestières sacrées.

\section{References:}

1. Alonga Longoma S. (2013). Étude microclimatique et pédologique congolaise: impact écologique de la fragmentation des écosystèmes. Cas des séries Yangambi et Yakonde à la région de Yangambi (R.D. Congo), Université Libre de Bruxelles, Faculté des Sciences, École Interfacultaire de Bioingénieurs, Service d’Écologie du paysage et Systèmes de Production Végétale, 2012-2013, 316p.

2. Alphandéry P. \& Fortier A. (2005). « Les savoirs locaux dans les dispositifs de gestion de la nature », Dans Bérard L., Cegarra M., Djama., Louafi S., Marchenay P., Roussel B. et Verdeaux F. (dir.), 
Biodiversité et savoirs naturalistes locaux en France, CIRAD, IDDRI, IFB, INRA, pp.158166,URL:http://www.fao.org/fileadmin/templates/olq/documents/doc uments/Biodiversityfrench.pdf, consulté le 10 janvier 2017.

3. Baco M.N., Biaou G., Pinton F. \& Lescure J.-P. (2007). « Les savoirs paysans traditionnels conservent-ils encore l'agrobiodiversité au Bénin ?», Biotechnol. Agron. Soc. Environ., Vol.11, n³, pp. 201210.

4. Baillargeon, D. (1993). " Histoire orale et histoire des femmes : itinéraires et points de rencontre»,Recherches féministes, vol. $6, \mathrm{n}^{\circ} 1$, pp. 53-68.URI: http://id.erudit.org/iderudit/057724ar, consulté le 21 avril 2016.

5. Beaulaton J.-D. \& Roussel B. (2005). «Les sites religieux Vodun : des patrimoines en permanente évolution ", Dans Cormier-SalemMC., D. Juhé-Beaulaton, J. Boutrais et B. Roussel (dir.),Patrimonialité de la nature tropicale. Dynamiques locales, enjeux internationaux. Paris, IRD, collection “Colloques et séminaires” :pp. 415-438.

6. Beaulaton, J.-D. (1999). " Arbres et bois sacrés : lieux de mémoire de l'ancienne Côte des Esclaves », Dans J.P. Chretien et J.L. Triaud (dir.), Histoire d'Afrique. Enjeux de mémoire; Paris, Karthala, 502 p.

7. Beguin, H. (1958). Géographie humaine de la région de Bengamisa, Publication de l’INEAC, série scientifique, $n^{\circ} 74,69 p$.

8. Bérard L., Cegarra M., Djama M., Louafi S., Marchenay P., Roussel B. \& Verdeaux F. (2005). « Savoirs et savoir-faire naturalistes locaux : l'originalité française ", VertigO - la revue électronique en sciences de l'environnement [En ligne], Volume 6 Numéro 1 | mai 2005, mis en ligne le 01 mai 2005, consulté le 05 mars 2016. URL : http://vertigo.revues.org/2887 ; DOI : 10.4000/vertigo.2887.

9. Boissière M. \& Doumenge C. (2008). «Entre marginalisation et démagogie : quelle place reste-t-il pour les communautés locales dans les aires protégées?", Les Cahiers d'Outre-Mer [En ligne], 244 | 2008, mis en ligne le 01 octobre 2011, consulté le 05 mars 2016, URL : http://com.revues.org/5476 ; DOI : 10.4000/com.5476.

10. Bossche J.V. (1954). Sectes et associations indigènes au Congo Belge, Éditions du bulletin militaire, Force publique, Léopoldville, 93p.

11. Boya Busquet B. (2006). «Des stratégies intégrées durables : savoir écologique traditionnel et gestion adaptative des espaces et des ressources", VertigO - la revue électronique en sciences de l'environnement [Online], Vol. 7, $\mathrm{n}^{\circ}$ 2, URL : http://vertigo.revues.org/2279 ; DOI : 10.4000/vertigo.2279. 
12. Crosnier C. (2005). « Les fruits de la préservation: le Parc national des Cévennes »,Dans Bérard L., Cegarra M., Djama., Louafi S., Marchenay P., Roussel B. et Verdeaux F. (dir.), Biodiversité et savoirs naturalistes locaux en France, CIRAD, IDDRI, IFB, INRA, pp.151-

158,URL:http://www.fao.org/fileadmin/templates/olq/documents/doc uments/Biodiversityfrench.pdf, consulté le 10 janvier 2017.

13. Donis, C. (1956). « La forêt dense congolaise et l'état actuel de sa sylviculture », Bulletin agricole du Congo belge, Vol. XLVII, $\mathrm{n}^{\circ} 2$, pp. 261-303.

14. Dupré, G. (1991). «Introduction », Dans Dupré G. (dir.), Savoirs paysans et développement. Paris : Karthala/ORSTOM,p. 17-35.

15. Ehinnou Koutchika R.I., Salako V.K., Tente B., Onodjè Agbani P., Chougourou D.C. 3, Boco Adjakpa J. \& SINSIN B. (2014). «Étude écologique des bois sacrés des Communes de Glazoué, Savè et Ouessè au Bénin», Journal of Animal \&Plant Sciences, Vol.21, Issue 3, pp.3313-3323, http://www.m.elewa.org/JAPS; consulté le 2 janvier 2017.

16. Esiso Asia-Amani F. (2012). Manuel de méthodologie de recherche en sciences sociales, PUK, IRSA, 171p.

17. Garcia, C., Pascal J.-P., Kushalappa C.G. (2006). « Les forêts sacrées du Kadagu en Inde: écologie et religion ", Bois et forêts des Tropiques, 288, 2, pp.5-13.

18. Gosse J.-P. (1963). « Le milieu aquatique et l'écologie des poissons dans la région de Yangambi », Annales du MRAC, série IN-8, Sciences zoologiques, $\mathrm{n}^{\circ} 116$, pp. 115- 270.

19. Grenand P. (1997). « L’ethnohistoire : une méthode de recherche pour les sociétés à tradition orale, l'exemple des Amérindiens de Guyane », Revue Equinoxe, n¹3, 1980, p. 47-58.

20. Halbwachs M. (1997). La mémoire collective, Rééditions Albin Michel, Paris, p. 295.

21. Henry J. (1951). Les bases théoriques des essais de paysannat indigène entrepris par l'INEAC au Congo belge, Bruxelles, 31p.

22. Henry J. (1979). «Les obstacles écologiques rencontrés lors des tentatives de rationalisation de l'agriculture traditionnelle des Turumbu », African, economic hystory, nº7, pp.155-170.

23. Hiergens T. (2010). «Un centre de recherche précieux enfoui sous des tonnes de poussière. À Yangambi subsiste l'espoir que tout redémarre un jour », Journal de la coopération belge, dimension 3, pp.19-20. 
24. Institut National de la Statistique (1984). Recensement scientifique de la population, Haut-Zaïre, Zones, Collectivités, Cités, Groupements, Quartiers, Kinshasa, 162p.

25. Juhé-Beaulaton D. (2003). «Processus de réactivation de sites sacrés dans le Sud du Bénin », Dans Gravari-Barbas M. \& Violier P. Lieux de culture, culture de lieux. Production(s) culturelle(s) locale(s) et émergence des lieux : dynamiques, acteurs, enjeux. Presses Universitaires de Rennes, pp. 67-79.

26. Juhé-Beaulaton D. (1999). «Arbres et bois sacrés : lieux de mémoire de l'ancienne Côte des Esclaves », Dans Chretien J.P. et Triaud J.L., Histoire d'Afrique. Enjeux de mémoire; Paris; Karthala, 502 p.

27. Jurion F. \& Henry J. (1967). De l'agriculture itinérante à l'agriculture intensifiée, INEAC, Hors-série, 435p.

28. Kigongo R. (2009). « 5. Les tombes Kasubi, matériaux Traditionnels et pratiques de conservation ", Dans Joffroy T. (dir.), Les pratiques de conservation traditionnelles en Afrique, ICCROM conservation studies, Rome, pp. 31-38.

29. Kombele Bishosha Menea (2004). Diagnostic de la fertilité des sols dans la cuvette centrale congolaise. Cas des séries Yangambi et Yakonde, Thèse en sciences agronomiques et ingénierie biologique, Faculté Universitaire des Sciences Agronomiques De Gembloux, 456p.

30. Koni Muluwa J. \& Bostoen K. (2008a). Noms et usages des plantes utiles chez les Nsong (RD Congo, Bandundu, bantu B85f). Gothembourg : University of Gothemburg,Department of Oriental and African Languages, Göteborg Africana Informal Series, $n^{\circ} 6$. En ligne:http://www.african.gu.se/downloads/gais06nsong.pdf, consulté le 10 novembre 2016.

31. Koni Muluwa J. et Bostoen K. (2008b). « Les plantes et l'invisible chez les Mbuun, Mpiin et Nsong (Bandundu, RD Congo) : une approche ethnolinguistique », Sprache und Geschichte in Afrika, $\mathrm{n}^{\circ} 2$, pp. 95-122.

32. Kouami Kokou et Sokpon N. (2006). « Les forêts sacrées du couloir du Dahomey », Bois et forêts des tropiques, Vol. 2, n 288, pp. 1523.

33. Louafi S. et Roussel B. (2005). «Introduction générale », Dans Bérard L., Cegarra M., Djama., Louafi S., Marchenay P., Roussel B. et Verdeaux F. (dir.), Biodiversité et savoirs naturalistes locaux en France, CIRAD, IDDRI, IFB, INRA, pp. 1315,URL:http://www.fao.org/fileadmin/templates/olq/documents/docu ments/Biodiversityfrench.pdf, consulté le 10 janvier 2017. 
34. Malan Djah F. (2009). « Religion traditionnelle et gestion durable des ressources floristiques en Cote d'Ivoire : Le cas des Ehotilé riverains du Parc national des îles Ehotilé », Vertigo-la revue électronique en science de l'environnement, volume 9, numéro 2, mis en ligne le 22 Septembre 2009, consulté le 27 Novembre 2016. URL : http ://vertigo.revues.org/8661 ; DOI :10.4000/vertigo.8661.

35. Malengreau G. (1952). «Les lotissements agricoles au Congo Belge », Bulletin Agricole du Congo Belge, n¹, vol.XLIII, pp.193242.

36. Marchenay P. (2005). «Conserver vivant, savoirs et pratiques locales : une gageure ? »,Dans Bérard L., Cegarra M., Djama., Louafi S., Marchenay P., Roussel B. et Verdeaux F. (dir.), Biodiversité et savoirs naturalistes locaux en France, CIRAD, IDDRI, IFB, INRA, pp.91-

98,URL:http://www.fao.org/fileadmin/templates/olq/documents/docu ments/Biodiversityfrench.pdf, consulté le 10 janvier 2017.

37. Moreau, A., Dedianne M-C., Letrilliart L., Le Goaziou M-F., Labarère J. \& Terra J.L. (2004). « S’approprier la méthode du focus group », La revue du praticien-médecine générale, 18, 645, pp. 382384.

38. Muller J. \& Vervier F. (1953). « Paysannat et coopérative Turumbu », Bulletin d'information de l'INEAC, vol.II, n², pp.115122.

39. Pinton F. \& Grenand P. (2007). « Savoirs traditionnels, populations locales et ressources globalisées », Les marchés de la biodiversité, pp.165-263.

40. Poll M. et Gosse J.-P. (1963). « Contribution à l'étude systématique de la faune ichthyologique du Congo central », Annales du MRAC, série IN-8, Sciences zoologiques, n 116, pp. 45-114.

41. Renard J., Henriot P., Schneider-Maunoury L. (2013). La gestion de la forêt de Mikea à Madagascar : comment concilier valeurs sociales, environnementales et économiques ? Atelier les valeurs de l'environnement : entre éthique et économie, Paris, Ceres-Erti, 16p.

42. Roué M. (2012). "Histoire et épistémologie des savoirs locaux et autochtones », Revue d'ethnoécologie[En ligne], 1 | 2012, mis en ligne le 02 décembre 2012, Consulté le 30 septembre 2016. URL : http:// ethnoecologie.revues.org/813 ; DOI : 10.4000/ethnoecologie.813.

43. Roussel B. (2005). «Savoirs locaux et conservation de la biodiversité : renforcer la représentation des communautés. ", Mouvements, vol. 4, $\quad \mathrm{n}^{\mathrm{0}}$ 41, pp. 82-88 URL : www.cairn.info/revue-mouvements-2005-4-page-82.htm. 
44. Savadogo S., Ouedraogo A. \& Thiombiano A. (2011). « Diversité et enjeux de conservation des bois sacrés en société Mossi (Burkina Faso) face aux mutations socioculturelles actuelles », Int. J. Biol. Chem. Sci., pp. 639-1658.

45. Sene C., Dioh P., \& Mormont M. (2013). « Pratiques traditionnelles et gestion durable des ressources naturelles : Etude de cas des sites naturels sacrés côtiers et marins à Jaol-Fadiouth», ASRDLF Appel à communication - Feuille de style, 18p.

46. Somé M. (2001-2002). «Les cultures africaines à l'épreuve de la colonisation», Afrika Zamani, n. 9 et 10, pp. 41-59.

47. Sow M. (2003) «Pratiques culturelles et conservation de la biodiversité en Guinée », Éducationrelative à l'environnement, Vol. 4, pp.289-295.

48. Staner P. (1955). «Les paysannats indigènes du Congo belge et du Ruanda-Urundi », Bulletinagricole du Congo belge, Vol. XLVI, N³, p.467-558.

49. Touré E.A. (2010). « Réflexion épistémologique sur l’usage des focus groups : fondements scientifiques et problèmes de scientificité », Dans Guillemette F., Luckerhoff J. et Baribeau C.,Entretiens de groupe : concepts, usages et ancrages, Recherches qualitatives, Vol. 29, n 1 , pp. 5-27.

50. Trudel F. (2002). « De l'ethnohistoire et l'histoire orale à la mémoire sociale chez les Inuits du Nunavut », Anthropologie et Sociétés, vol. 26, $\quad \mathrm{n}^{\circ} 2-3, \quad$ 2002, 2 . 137-159. URI: http://id.erudit.org/iderudit/007052ar, consulté le 21 avril 2016.

51. Tubiana L. (2005). « Les savoirs naturalistes locaux, une question globale », Dans Bérard L., Cegarra M., Djama., Louafi S., Marchenay P., Roussel B. \& Verdeaux F. (dir.) (2017). Biodiversité et savoirs naturalistes locaux en France, CIRAD, IDDRI, IFB, INRA, pp. 78,URL:http://www.fao.org/fileadmin/templates/olq/documents/docu ments/Biodiversityfrench.pdf, consulté le 10 janvier.

52. Van Wambeke, A. \& Evrard C. (1954). Carte du sol et de la végétation du Congo belge et du Ruanda-Urundi, 6. Yangambi planchette 1: Weko $A$ et $B$, Notice explicative de la carte des sols et de la végétation, INEAC, Bruxelles, 23p.

53. Vansina J. (1987). «Vers une histoire des sociétés Mongo », Annales Aequatoria, 8, pp.9-57.

54. Yameogo L. (2015). «Le patrimoine méconnu des bois sacrés de la ville de Koudougou (Burkina Faso) : de la reconnaissance à la sauvegarde», Cahiers de géographie du Québec, Vol.59, n 166, pp. 71-90. 


\section{Documents d'archives}

1. Brandt L. (1917). "Le mambela en territoire de Bafwasende », Archives africaines du Ministère Belge des Affaires Etrangères, commerce extérieur et coopération au développement, AIMO, Portefeuille $\mathrm{n}^{\circ} 1587$.

2. Coenen Ph. (1937) «De la psychose Kitawala », Archives du Musée Royal d'Afrique Centrale, Section d'Histoire du Temps présent.

3. Colinet P. (1942). « Rapport $n^{\circ} 2$ sur les agissements des relégués de la secte Kitawala en territoire de Lubutu », Archives du Musée Royal d'Afrique Centrale, Section d'Histoire du Temps présent, Lubutu.

4. Colinet P. (1942). « Rapport $\mathrm{n}^{\circ} 3$ sur la diffusion de la secte Kitawala en Territoire de Lubutu ", Archives du Musée Royal d'Afrique Centrale, Section d'Histoire du Temps présent, Lubutu.

5. Cote P. (1939). « Note au sujet du mouvement Kitawala ou Watch Tower en région de Kwamouth", Archives du Musée Royal d'Afrique Centrale, Section d'Histoire du Temps présent, Kwamouth.

6. De Valck (1938). « Notes sur les agissements des relégués adeptes du Kitawala à Bosolo », Archives du Musée Royal d'Afrique Centrale, Section d'Histoire du Temps présent, Bosolo.

7. Demptine, SD. Histoire de la Tribu des Turumbu, Archives africaines du Ministère Belge des Affaires Etrangères, commerce extérieur et coopération au développement, AIMO, Portefeuille $n^{\circ} 1587$, Liasse $n^{\circ} 68$.

8. Gilot P. (1947). «Le Kitawala en Territoire de Lubutu », Archives du Musée Royal d'Afrique Centrale, Section d'Histoire du Temps présent, Lubutu.

9. Laurent J. (1937). « Procès-verbal de Conseil des notables du 24 octobre 1937, Chefferie Yaelongo ", Archives de la Division Provinciale de l'Intérieur, Sécurité et Affaires coutumières de la Province de la Tshopo, Dossier AIMO.

10. Lauwers Y. (1932a). " Notes ethnographiques des peuplades des Bambole ", Archives africaines du Ministère Belge des Affaires Etrangères, commerce extérieur et coopération au développement, AIMO, Portefeuille $n^{\circ} 1578$, Liasse $n^{\circ} 28$.

11. Lauwers Y. (1932b). "Annexe aux rapports d'enquête, Chefferies Yelongo et Yawenda", Archivesde la Division Provinciale de l'Intérieur, Sécurité et Affaires coutumières de la Province de la Tshopo, Dossier AIMO.

12. Libert N. (1936). " Procès-verbal du Conseil de Chefferie tenu au village Yaselia, le 14 juillet 1936 », Archives du Musée Royal d'Afrique Centrale, Section d'Histoire du Temps présent, Yaselia. 
13. Marmitte (1922). « Note sur la migration des Bambole », Archives africaines du Ministère Belge des Affaires Étrangères, commerce extérieur et coopération au développement, AIMO, Portefeuille $\mathrm{n}^{\circ} 1578$, Liasse $\mathrm{n}^{\circ} 28$.

14. Rapport AIMO (1933). «Territoire de Banalia», Archives du Musée Royal d'Afrique Centrale, Section d'Histoire du Temps présent.

15. Rapport AIMO (1934). «Territoire de Topoke-Lokele, Isangi », Archives du Musée Royal d'Afrique Centrale, Section d'Histoire du Temps présent.

16. Rapport AIMO (1933). «Tableau synoptique de la population du Territoire de Topoke-Lokele, Isangi», Archives de la Division Provinciale de l'Intérieur, Sécurité et Affaires coutumières de la Province de la Tshopo, Dossier AIMO.

17. INEAC (1939). Rapport annuel pour l'exercice 1939, hors série 1940 et 1941.

18. Schollar A. (1944). «Rapport d'instruction au sujet de la révolte Kitawala en Territoire de Lubutu en mars 1944 », Archives du Musée Royal d'Afrique Centrale, Section d'Histoire du Temps présent, Lubutu.

19. Secteur Bamanga (2015). Rapport annuel, Exercice 2015, Tableau synoptique de recensement de la population congolaise, Bengamisa.

20. Secteur Turumbu (2015). Rapport de premier trimestre, Exercice 2015. Tableau synoptique de recensement de la population congolaise, Yaselia.

21. Steens M. (1934). «Lettre du $1^{\text {er }}$ juillet 1934 adressée à l'Administrateur du Territoire d'Isangi ", Archives du Musée Royal d'Afrique Centrale, Section d'Histoire du Temps présent.

22. Van Dieren, M. (1933). « Circoncision chez les Bamanga », Archives africaines du Ministère Belge des Affaires Étrangères, commerce extérieur et coopération au développement, AIMO, Portefeuille $n^{\circ} 1587$, Liasse $n^{\circ} 71$. 